\title{
Association between Hemoglobin Levels and Colorectal Polyps in Asymptomatic Chinese Adults
}

\author{
Chao Shen, ${ }^{1}$ Xiaoying Shi, ${ }^{2}$ Maram Al-Ashoor, ${ }^{2}$ and Chengfu Xu $\mathbb{D}^{2}$ \\ ${ }^{1}$ Health Management Center, The First Affiliated Hospital, College of Medicine, Zhejiang University, Hangzhou 310003, China \\ ${ }^{2}$ Department of Gastroenterology, The First Affiliated Hospital, College of Medicine, Zhejiang University, Hangzhou 310003, China
}

Correspondence should be addressed to Chengfu Xu; xiaofu@zju.edu.cn

Received 18 November 2019; Revised 1 January 2020; Accepted 11 January 2020; Published 18 January 2020

Academic Editor: Vicent Hernández

Copyright (c) 2020 Chao Shen et al. This is an open access article distributed under the Creative Commons Attribution License, which permits unrestricted use, distribution, and reproduction in any medium, provided the original work is properly cited.

\begin{abstract}
Background/Aims. Metabolic and hematologic aberrations are often observed in individuals with colorectal polyps. This study was aimed at investigating whether hemoglobin levels are associated with colorectal polyps in asymptomatic Chinese adults. Methods. A cross-sectional analysis was performed among 1633 asymptomatic adults who underwent colonoscopy examinations during routine health check-ups at the First Affiliated Hospital, College of Medicine, Zhejiang University, between 2015 and 2018. Results. A total of 449 (27.50\%) participants were diagnosed with colorectal polyps, and those with colorectal polyps had significantly higher hemoglobin levels than did those without colorectal polyps. Hemoglobin levels were positively associated with the prevalence of colorectal polyps, with rates of $16.43 \%, 26.20 \%, 32.17 \%$, and $35.87 \%$ among participants with hemoglobin levels in the first, second, third, and fourth quartiles, respectively $(P$ for trend $<0.001)$. Stepwise multiple regression analysis showed that elevated hemoglobin levels independently increased the risk of colorectal polyps (odd ratio $=1.017 ; 95 \%$ confidence interval: 1.008-1.026). Conclusion. Elevated hemoglobin levels were significantly and independently associated with the prevalence and risk of colorectal polyps in asymptomatic adults.
\end{abstract}

\section{Introduction}

Colorectal cancer (CRC) is ranked as the second most common cancer diagnosed in women and the third most common cancer in men worldwide [1]. The incidence and mortality of CRC have shown an increasing trend in China over the past decade [2]. This trend can be attributed to many factors, including rapid urbanization, an aging population, and a shift toward a more sedentary lifestyle and unhealthy dietary habits [2].

It has been well established that most CRCs develop from adenomatous polyps. The pathogenesis of CRC has been extensively studied, and most invasive CRCs develop from adenomas following the adenoma-carcinoma sequence [3]. In general, CRCs that arise sporadically result from the stepwise accumulation of multiple somatic mutations [4]. This hypothesis is supported by pathological, epidemiological, and observational clinical data, and it is widely accepted that early detection and resection of these polyps may prevent CRC-related death [5]. Multiple CRC screening guidelines have been issued. Although recommendations vary, they are commonly based on the individual's age and risk factors [6]. However, due to certain limitations, such as the availability of national screening programs and medical resources, CRC screening in China is less likely to be conducted in the average-risk population [7]. Identifying risk factors and associations of certain inexpensive laboratory parameters can be useful in targeting groups of people for colonoscopy who are generally regarded as average-risk individuals.

A simple blood test, which is normally ordered during an annual routine health screening, can detect certain aberrations in laboratory parameters. These aberrations may be utilized to help physicians identify and stratify populations with high incidences of colorectal polyps or CRC. Kinar et al. reported that the information contained in the complete blood count report could be processed automatically by a machine learning flagging system to identify individuals with high risk of CRC [8]. In addition, hemoglobin levels have been shown to be elevated in metabolic diseases that are closely associated with colorectal polyps, such as metabolic 
syndrome and nonalcoholic fatty liver disease [9-12]. Chung et al. reported that an elevated hemoglobin level is associated with an increased risk of developing metabolic syndrome [9]. Our previous study, along with other studies, also found that hemoglobin levels are positively associated with both the prevalence and incidence of nonalcoholic fatty liver disease $[10,11]$, and elevated glycosylated hemoglobin levels are useful in predicting adenomatous polyps [13]. These hematologic and metabolic parameters can readily be obtained through inexpensive routine screening tests, and identifying associations between certain laboratory parameters and the risk of colorectal polyps may be of great use in the early detection of colorectal polyps.

In this study, we aimed to investigate whether hemoglobin levels are associated with the prevalence and risk of colorectal polyps in average-risk asymptomatic adults.

\section{Materials}

2.1. Study Population. This cross-sectional study was performed among asymptomatic adults who underwent colonoscopy examinations during routine health check-ups at the First Affiliated Hospital, College of Medicine, Zhejiang University, between 2015 and 2018. Because we mainly focused on the average-risk screening population, the following participants were excluded: those with a personal history of colonic diseases (colorectal cancer, colorectal polyps, and inflammatory bowel disease), a history of colorectal surgery, or a family history of colorectal cancer or hereditary cancer syndrome. We also excluded patients who underwent colonoscopies that did not reach the cecum and those with inadequate bowel preparation (Boston Bowel Preparation Scale score less than six). A total of 1633 participants (1123 men and 510 women) were enrolled in this study. The study protocol was approved by the hospital ethics committee.

2.2. Clinical Evaluations. Trained physicians performed the clinical evaluations according to standardized procedures $[14,15]$. Standing height, waist circumference, and body weight without shoes and with light clothes were recorded in the morning using standard procedures. The body mass index (BMI) was calculated as the body weight (kg) divided by the height squared (meters). Blood pressure was measured in the sitting position after $10 \mathrm{~min}$ of rest using an automated sphygmomanometer following a standard protocol.

A fasting blood sample was obtained from each participant for analysis of blood hemoglobin level and serum biochemical variables. The blood hemoglobin level was analyzed using an automated hematology analyzer (Sysmex XT-1800, Kobe, Japan) according to the manufacturer's instructions. Serum biochemical variables were measured using a Hitachi 7600 autoanalyzer (Hitachi, Tokyo, Japan) with standard methods.

2.3. Colonoscopy Examination. Experienced endoscopists performed colonoscopies after the bowel was adequately prepared. The preparation included self-administration of 2-4 L of polyethylene glycol solution six hours before the colonoscopy. The endoscopists determined the adequacy of the bowel preparation using the Boston Bowel Preparation Scale (BBPS). A successful colonoscopy was defined as one with cecal intubation, which was confirmed by photography. The size, number, and location of polyps were recorded by the endoscopists for further analysis. Those with a successful colonoscopy and a BBPS score of six or higher were included in the final analysis.

2.4. Statistical Analysis. Statistical analyses were performed using SPSS 18.0 software for Windows (SPSS Inc., Chicago, IL). Quantitative variables are expressed as the mean and standard deviation for normally distributed variables or median and interquartile range for nonnormally distributed variables. Student's $t$-tests, Mann-Whitney $U$ tests, or chisquare tests were applied to compare variables. Multiple stepwise regression analysis (backward: Wald; cutoff for entry: 0.05; cutoff for removal: 0.10) was performed to explore risk factors for colorectal polyps. $P$ values less than 0.05 (2-tailed) were considered to indicate statistical significance.

\section{Results}

3.1. Hemoglobin Levels Are Elevated in Participants with Colorectal Polyps. Among the 1633 participants included in the analysis of this study, 449 (27.50\%) were diagnosed with colorectal polyps; three participants were found to have histologically confirmed colorectal carcinoma. Among those with polyps, $267(59.47 \%)$ participants had a single polyp and $182(40.53 \%)$ had two or more. Most of the participants $(n=300,66.82 \%)$ had left-sided polyps, $97(21.60 \%)$ had right-sided polyps, and 52 (11.58\%) had both-sided polyps. The size of polyps ranged from $2.0 \mathrm{~mm}$ to $40.0 \mathrm{~mm}$ with a medium (interquartile range) of $4.0 \mathrm{~mm}(2.0-6.0 \mathrm{~mm})$. For 232 participants, biopsies or resections of colorectal polyps were performed; 139 (59.91\%) were identified as having neoplastic polyps and $93(40.09 \%)$ as having nonneoplastic polyps. Clinical characteristics were compared between those with and those without colorectal polyps (Table 1).

The participants with colorectal polyps were older, predominantly male, and had a greater waist circumference, a higher BMI, and a higher blood pressure than those without colorectal polyps (Table 1). Those with colorectal polyps also had significantly higher serum levels of alanine aminotransferase (ALT), aspartate aminotransferase (AST), $\gamma$ glutamyltransferase (GGT), triglycerides, uric acid, and carcinoembryonic antigen (CEA), as well as higher total and LDL cholesterol and fasting blood glucose, than those without colorectal polyps. In addition, serum HDL cholesterol levels in participants with colorectal polyps were lower than those in participants without colorectal polyps (all with $P<0.01$, Table 1). These results indicate that participants with colorectal polyps had worse metabolic profiles than those without colorectal polyps. Another interesting finding is that hemoglobin levels were significantly higher in participants with colorectal polyps than in those without colorectal polyps (Table 1). We also found that hemoglobin levels tended to be higher in participants with neoplastic polyps than in those with nonneoplastic polyps $(152.3 \pm 13.3 \mathrm{~g} / \mathrm{L}$ vs. $149.3 \pm 15.2 \mathrm{~g} / \mathrm{L}, P=0.111)$. These findings indicated that 
TABLE 1: Characteristics of study participants with and without colorectal polyps.

\begin{tabular}{|c|c|c|c|c|}
\hline Variables & With colorectal polyps & Without colorectal polyps & $t$ value & $P$ value \\
\hline$n$ (male/female) & $449(359 / 90)$ & $1184(764 / 420)$ & $36.081^{\mathrm{a}}$ & $<0.001$ \\
\hline Age (years) & $52.1(8.7)$ & $49.1(9.4)$ & 5.925 & $<0.001$ \\
\hline Waist circumference $(\mathrm{cm})$ & $89.1(9.1)$ & $85.5(9.2)$ & 6.993 & $<0.001$ \\
\hline Body mass index $\left(\mathrm{kg} / \mathrm{m}^{2}\right)$ & $25.09(3.18)$ & $24.18(3.18)$ & 5.157 & $<0.001$ \\
\hline Systolic blood pressure $(\mathrm{mmHg})$ & $130.4(16.7)$ & $126.9(17.7)$ & 3.639 & $<0.001$ \\
\hline Diastolic blood pressure (mmHg) & $79.5(10.8)$ & $77.1(11.8)$ & 3.711 & $<0.001$ \\
\hline Alanine aminotransferase (U/L) & $22.0(16.0-30.0)$ & $19.0(14.0-28.0)$ & $4.171^{\mathrm{b}}$ & $<0.001$ \\
\hline Aspartate aminotransferase (U/L) & $21.0(18.0-25.0)$ & $20.0(17.0-25.0)$ & $2.641^{\mathrm{b}}$ & 0.008 \\
\hline$\gamma$-Glutamyltransferase $(\mathrm{U} / \mathrm{L})$ & $32.0(21.0-54.0)$ & $25.0(16.0-44.0)$ & $5.647^{\mathrm{b}}$ & $<0.001$ \\
\hline Triglyceride (mmol/L) & $1.64(1.16-2.43)$ & $1.42(0.99-2.11)$ & $4.825^{\mathrm{b}}$ & $<0.001$ \\
\hline Total cholesterol (mmol/L) & $4.78(4.25-5.43)$ & $4.61(4.08-5.24)$ & $3.376^{\mathrm{b}}$ & 0.001 \\
\hline HDL cholesterol (mmol/L) & $1.13(0.93-1.33)$ & $1.20(1.00-1.44)$ & $4.058^{\mathrm{b}}$ & $<0.001$ \\
\hline LDL cholesterol (mmol/L) & $2.75(2.27-3.30)$ & $2.61(2.17-3.14)$ & $3.169^{\mathrm{b}}$ & 0.002 \\
\hline Fasting plasma glucose $(\mathrm{mmol} / \mathrm{L})$ & $4.85(4.50-5.35)$ & $4.78(4.41-5.19)$ & $2.975^{\mathrm{b}}$ & 0.003 \\
\hline Serum uric acid $(\mu \mathrm{mol} / \mathrm{L})$ & $366.3(85.4)$ & $343.1(88.1)$ & 4.779 & $<0.001$ \\
\hline Carcinoembryonic antigen $(\mu \mathrm{g} / \mathrm{L})$ & $2.00(1.50-2.90)$ & $1.80(1.30-2.60)$ & $5.001^{\mathrm{b}}$ & $<0.001$ \\
\hline Hemoglobin (g/L) & $151.9(14.7)$ & $145.9(16.4)$ & 6.795 & $<0.001$ \\
\hline
\end{tabular}

Data are expressed as the mean (SD) or median (IQR). ${ }^{a} \chi^{2}$ value. ${ }^{\mathrm{b}} Z$ value. HDL: high-density lipoprotein; LDL: low-density lipoprotein.

Table 2: Association between hemoglobin level and the prevalence of colorectal polyps.

\begin{tabular}{lccccccc}
\hline Hemoglobin & Total & $\begin{array}{c}\text { Colorectal } \\
\text { polyps }\end{array}$ & PR\% & PR & $\chi^{2}$ & $P$ \\
\hline Quartile 1 & 414 & 68 & 16.43 & 1.00 & & \\
Quartile 2 & 439 & 115 & 26.20 & 1.59 & & \\
Quartile 3 & 373 & 120 & 32.17 & 1.96 & & \\
Quartile 4 & 407 & 146 & 35.87 & 2.18 & 44.240 & $<0.001$ \\
\hline
\end{tabular}

PR\%: prevalence rate; PR: prevalence ratio.

hemoglobin levels are potentially associated with colorectal polyps, and the levels may have a stronger association with neoplastic polyps than with nonneoplastic polyps.

3.2. Hemoglobin Levels Are Positively Associated with the Prevalence of Colorectal Polyps. To further explore the association between hemoglobin level and colorectal polyps, we divided all participants into quartiles according to their hemoglobin level: quartile 1 , hemoglobin $\leq 136 \mathrm{~g} / \mathrm{L}$; quartile $2,136 \mathrm{~g} / \mathrm{L}<$ hemoglobin $\leq 150 \mathrm{~g} / \mathrm{L}$; quartile $3,150 \mathrm{~g} / \mathrm{L}<$ hemoglobin $\leq 159 \mathrm{~g} / \mathrm{L}$; and quartile 4 , hemoglobin $>159 \mathrm{~g} / \mathrm{L}$. We observed the hemoglobin quartiles to be positively correlated with the prevalence of colorectal polyps. The prevalence of colorectal polyps was $16.43 \%$ among the participants with hemoglobin levels in the first quartile, and the prevalence increased to $26.20 \%, 32.17 \%$, and $35.87 \%$ in the second, third, and fourth quartiles, respectively ( $P$ for trend $<0.001$, Table 2). This finding shows that individuals who have higher hemoglobin levels are more likely to have colorectal polyps.

3.3. Hemoglobin Levels Are Associated with Colorectal Polyps Independent of Obesity. Obesity is a major risk factor for CRC and a potential cofactor for the association between hemoglobin level and colorectal polyps. To explore whether this association is independent of obesity, we excluded 680 participants who were overweight/obese (BMI $\left.\geq 25 \mathrm{~kg} / \mathrm{m}^{2}\right)$. Of the remaining 953 lean participants, $228(23.92 \%)$ had colorectal polyps. Hemoglobin levels remained significantly higher in the participants with colorectal polyps than in those without colorectal polyps (148.8 (15.7) g/L vs. 142.5 (16.8) g/L, $P<$ $0.001)$; thus, a positive correlation between hemoglobin levels and the prevalence of colorectal polyps was observed among lean participants. The prevalence of colorectal polyps was $15.43 \%, 24.24 \%, 30.37 \%$, and $32.18 \%$ in quartiles $1,2,3$, and 4 , respectively $(P$ for trend $<0.001)$. The results of this subgroup analysis show that the association of hemoglobin levels with colorectal polyps is independent of obesity.

3.4. Elevated Hemoglobin Levels Independently Increase the Risk of Colorectal Polyps. We also conducted stepwise multiple regression analysis to investigate the association of hemoglobin levels with the risk of colorectal polyps. Seventeen variables, including age, sex, waist circumference, BMI, systolic and diastolic blood pressure, ALT level, AST level, GGT level, triglyceride level, total cholesterol, HDL cholesterol, LDL cholesterol, fasting blood sugar, serum uric acid level, CEA level, and hemoglobin level, were input into the regression equation. We found that six variables remained in the final equation, suggesting them to be independently associated with the risk of colorectal polyps (Table 3). An important finding was that elevated hemoglobin levels were significantly associated with an increased risk of colorectal polyps (OR $=1.017 ; 95 \% \mathrm{CI}$ : 1.008-1.026). This finding further supports a significant association between hemoglobin level and colorectal polyps. 
TABLE 3: Risk factors associated with the presence of colorectal polyps.

\begin{tabular}{lccccc}
\hline Variables & $\beta$ & SE & Wald $\chi^{2}$ & $P$ & OR $(95 \%$ CI $)$ \\
\hline Age (years) & 0.037 & 0.007 & 29.148 & $<0.001$ & $1.038(1.024-1.052)$ \\
Waist circumference $(\mathrm{cm})$ & 0.020 & 0.008 & 6.651 & 0.010 & $1.020(1.005-1.035)$ \\
Total cholesterol $(\mathrm{mmol} / \mathrm{L})$ & 0.142 & 0.067 & 4.435 & 0.035 & $1.152(1.010-1.314)$ \\
HDL cholesterol $(\mathrm{mmol} / \mathrm{L})$ & -0.476 & 0.204 & 5.445 & 0.020 & $0.622(0.417-0.927)$ \\
Carcinoembryonic antigen $(\mu \mathrm{g} / \mathrm{L})$ & 0.094 & 0.033 & 7.947 & 0.005 & $1.099(1.029-1.173)$ \\
Hemoglobin $(\mathrm{g} / \mathrm{L})$ & 0.017 & 0.004 & 14.343 & $<0.001$ & $1.017(1.008-1.026)$ \\
\hline
\end{tabular}

$\beta$ : partial regression coefficient; SE: standard error of the partial regression coefficient; OR: odds ratio; CI: confidence interval; HDL: high-density lipoprotein.

\section{Discussion}

In this study, we found a significant positive association between hemoglobin levels and colorectal polyps. Hemoglobin levels were significantly higher in participants with colorectal polyps than in those without colorectal polyps. Hemoglobin levels were positively associated with the prevalence of colorectal polyps, and this association was independent of obesity. Our results also showed that elevated hemoglobin independently increased the risk of colorectal polyps. These findings may have potential clinical significance.

Currently, screening for CRC is not widely available for average-risk asymptomatic adults in many rural communities and large populations. Indeed, China serves as an example of a country with a large population and a great disparity in medical resources, and national screening programs for colorectal polyps and cancer have yet to be implemented. This delay might be attributed to the large population and the strain a screening program may place on the limited medical resources of the country [2]. Therefore, developing a more comprehensive risk assessment for asymptomatic average-risk adults may be beneficial for targeting those who are at relatively high risk and are recommended to undergo more invasive procedures, such as a colonoscopy [16].

Screening recommendations for classically described average-risk adults might be amended in areas with limited available resources; even the most effective and easy-touse screening test, the fecal immunochemical test, may not be sufficient for detecting early adenomas [17]. Therefore, if the goal is to decrease the CRC burden, total colonoscopy is the best option for screening [5]. However, due to the increasingly aging population in China, it is almost impossible to apply the agreed-upon recommendations for screening asymptomatic adults. In addition, data on the cost-effectiveness of this strategy for these large populations are limited. One solution would be to find potential markers that may indicate the presence of colorectal polyps.

Studies have developed scoring systems that may help physicians easily stratify asymptomatic adults based on risk factors and direct them for colonoscopy [18]. This strategy might be beneficial for the identification of at-risk individuals in targeted areas and populations with limited resources as well as for scheduling of colonoscopy screening at tertiary hospitals. The application of this scheme would theoretically allow countries to increase screening and decrease the colorectal cancer burden while realistically considering the availability of medical resources.
Colorectal polyps are closely associated with metabolic diseases, such as metabolic syndrome and nonalcoholic fatty liver disease [19, 20], and several studies have reported a strong positive association of elevated hemoglobin with these conditions [9-11, 21]. This association may implicate a high hemoglobin level as a major factor in several pathologies and disorders. In this study, we focused on the association between high hemoglobin levels and colorectal polyps, and our results suggested that elevated hemoglobin may serve as an easily obtained indicator for colorectal polyps. This marker may allow the creation of a novel scoring system for predicting the presence of colorectal polyps and identifying early lesions for colonoscopy and resection.

Although the mechanism linking high hemoglobin levels and colorectal polyps is unclear, there are several probable explanations. One possible reason is that the presence of high levels of hemoglobin, an iron-containing metalloprotein, causes iron-induced oxidative stress and relative antioxidant depletion; this process has been shown to promote carcinogenesis $[22,23]$. This hypothesis may support the possibility that elevated hemoglobin levels act as additive factors in the formation of colonic adenomas and their transformation into carcinomas via the adenoma-carcinoma sequence. Further clarification of the underlying mechanism will help in understanding the relationship between hemoglobin and colorectal polyps.

The strength of this study lies in the large target population of asymptomatic adults and the evaluation of different serum parameters and anthropometric measurements, which were employed to assess correlations between changes in certain parameters and the presence of colorectal polyps during screening colonoscopy. The clearly observed direct proportional association between hemoglobin level and colorectal polyps is a promising avenue for further studies to analyze the existence of a causal relationship between elevated hemoglobin and the incidence of colorectal polyps. In addition, more investigations into the pathophysiological process should be conducted.

Several limitations also exist in our study, including a lack of data regarding the dietary and smoking habits of the examined population. Possible confounding factors affecting hemoglobin levels, including smoking and increased red meat intake, might also be associated with the development of colorectal polyps [24, 25]. These confounding factors should be taken into consideration in future studies. The second limitation is that the estimated adenoma detection rate is relatively low in this study. A possible explanation is that 
almost half of the participants (794/1633) were younger than 50 years of age in this study. Young adults may have a lower adenoma detection rate than old ones. Moreover, histological diagnosis of the colorectal polyps found during the colonoscopies was not obtained in all cases, and different risk potentials exist for cancer progression among the different types of colorectal polyps [26]. Although we found that hemoglobin levels tended to be higher in participants with neoplastic polyps than in those with nonneoplastic polyps, further studies are necessary to confirm the association between hemoglobin levels and different types of colorectal polyps.

In conclusion, on the basis of this large colonoscopybased study, we found a direct proportional association between hemoglobin level and colorectal polyps in an average-risk asymptomatic Chinese adult population. Further prospective studies should be performed to clarify these findings and identify any causal relationships between hemoglobin level and colorectal polyps.

\section{Data Availability}

Association between hemoglobin levels and colorectal polyps in asymptomatic Chinese adults.

\section{Conflicts of Interest}

The authors declare no conflict of interest.

\section{Authors' Contributions}

CS did the statistical analysis, data collection and interpretation, and manuscript writing. CS, XYS, and MA did the data collection and interpretation and manuscript writing. CFX did the study design and implementation, manuscript drafting, and critical revision of the manuscript for important intellectual content. All authors read and approved the final manuscript.

\section{Acknowledgments}

The authors thank the editors of AJE (https://www.aje.com) for their assistance with the English grammar of the manuscript (Certificate Verification Key: 6179-7B03-C9F0-A75A04B0).

\section{References}

[1] F. Bray, J. Ferlay, I. Soerjomataram, R. L. Siegel, L. A. Torre, and A. Jemal, "Global cancer statistics 2018: GLOBOCAN estimates of incidence and mortality worldwide for 36 cancers in 185 countries," CA: a Cancer Journal for Clinicians, vol. 68, no. 6, pp. 394-424, 2018.

[2] L. Xi, J. Zhu, H. Zhang, M. Muktiali, C. Xu, and A. Wu, "Epidemiological trends in gastrointestinal cancers in China: an ecological study," Digestive Diseases and Sciences, vol. 64, no. 2, pp. 532-543, 2019.

[3] V. Aran, A. P. Victorino, L. C. Thuler, and C. G. Ferreira, "Colorectal cancer: epidemiology, disease mechanisms and interventions to reduce onset and mortality," Clinical Colorectal Cancer, vol. 15, no. 3, pp. 195-203, 2016.
[4] K. Tariq and K. Ghias, "Colorectal cancer carcinogenesis: a review of mechanisms," Cancer Biology \& Medicine, vol. 13, no. 1, pp. 120-135, 2016.

[5] A. G. Zauber, S. J. Winawer, M. J. O'Brien et al., "Colonoscopic polypectomy and long-term prevention of colorectal-cancer deaths," The New England Journal of Medicine, vol. 366, no. 8, pp. 687-696, 2012.

[6] R. A. Smith, K. S. Andrews, D. Brooks et al., "Cancer screening in the United States, 2018: a review of current American Cancer Society guidelines and current issues in cancer screening," CA: a Cancer Journal for Clinicians, vol. 68, no. 4, pp. 297-316, 2018.

[7] D. Y. Leung, K. M. Chow, S. W. Lo, W. So, and C. Chan, "Contributing factors to colorectal cancer screening among Chinese people: a review of quantitative studies," International Journal of Environmental Research and Public Health, vol. 13, no. 5, p. 506, 2016.

[8] Y. Kinar, P. Akiva, E. Choman et al., "Performance analysis of a machine learning flagging system used to identify a group of individuals at a high risk for colorectal cancer," PLoS One, vol. 12, article e0171759, 2017.

[9] G. E. Chung, J. Y. Yim, D. Kim et al., “Associations between hemoglobin concentrations and the development of incidental metabolic syndrome or nonalcoholic fatty liver disease," Digestive and Liver Disease, vol. 49, no. 1, pp. 57-62, 2017.

[10] L. Xu, C. F. Xu, C. H. Yu, M. Miao, and Y. M. Li, "Haemoglobin and non-alcoholic fatty liver disease: further evidence from a population-based study," Gut, vol. 58, no. 12, pp. 1706-1707, 2009.

[11] C. Yu, C. Xu, L. Xu, J. Yu, M. Miao, and Y. Li, "Serum proteomic analysis revealed diagnostic value of hemoglobin for nonalcoholic fatty liver disease," Journal of Hepatology, vol. 56, no. 1, pp. 241-247, 2012.

[12] A. Tanoglu and M. Kara, "Nonalcoholic fatty liver diseaserelated cardiovascular risk: is there an association with blood hemoglobin levels?," European Journal of Gastroenterology \& Hepatology, vol. 27, no. 10, pp. 1126-1129, 2015.

[13] B. J. Kim, Y. H. Kim, D. H. Sinn et al., "Clinical usefulness of glycosylated hemoglobin as a predictor of adenomatous polyps in the colorectum of middle-aged males," Cancer Causes of Control, vol. 21, no. 6, pp. 939-944, 2010.

[14] J. Wang, L. Ma, S. Chen et al., "Risk for the development of non-alcoholic fatty liver disease: a prospective study," Journal of Gastroenterology and Hepatology, vol. 33, no. 8, pp. 1518$1523,2018$.

[15] C. Xu, M. Yan, Y. Sun et al., "Prevalence of Helicobacter pylori infection and its relation with body mass index in a Chinese population," Helicobacter, vol. 19, no. 6, pp. 437442, 2014.

[16] H. Chen, N. Li, J. Ren et al., "Participation and yield of a population-based colorectal cancer screening programme in China," Gut, vol. 68, no. 8, pp. 1450-1457, 2019.

[17] H. M. Chiu, J. Y. Ching, K. C. Wu et al., "A risk-scoring system combined with a fecal immunochemical test is effective in screening high-risk subjects for early colonoscopy to detect advanced colorectal neoplasms," Gastroenterology, vol. 150, no. 3, pp. 617-625.e3, 2016.

[18] T. Ohno, S. Adachi, M. Okuno et al., "Development of a novel scoring system for predicting the risk of colorectal neoplasia: a retrospective study," PLoS One, vol. 11, no. 6, article e0157269, 2016. 
[19] S. Tal, E. Melzer, T. Chsherbakov, and S. Malnick, "Metabolic syndrome is associated with increased prevalence of advanced colorectal polyps," The Journal of Nutrition, Health \& Aging, vol. 18, no. 1, pp. 22-25, 2014.

[20] V. W. Wong, G. L. Wong, S. W. Tsang et al., "High prevalence of colorectal neoplasm in patients with non-alcoholic steatohepatitis," Gut, vol. 60, no. 6, pp. 829-836, 2011.

[21] Y. Hashimoto, M. Tanaka, T. Kimura et al., "Hemoglobin concentration and incident metabolic syndrome: a populationbased large-scale cohort study," Endocrine, vol. 50, no. 2, pp. 390-396, 2015.

[22] N. Adris, A. C. G. Chua, M. W. Knuiman, M. L. Divitini, D. Trinder, and J. K. Olynyk, "A prospective cohort examination of haematological parameters in relation to cancer death and incidence: the Busselton Health Study," BMC Cancer, vol. 18, no. 1, p. 863, 2018.

[23] A. Sonmez, M. I. Yilmaz, M. Saglam et al., "The relationship between hemoglobin levels and endothelial functions in diabetes mellitus," Clinical Journal of the American Society of Nephrology, vol. 5, no. 1, pp. 45-50, 2010.

[24] P. J. Hu, S. H. Ley, S. N. Bhupathiraju, Y. Li, and D. D. Wang, "Associations of dietary, lifestyle, and sociodemographic factors with iron status in Chinese adults: a cross-sectional study in the China Health and Nutrition Survey," The American Journal of Clinical Nutrition, vol. 105, no. 2, pp. 503-512, 2017.

[25] M. Malenica, B. Prnjavorac, T. Bego et al., "Effect of cigarette smoking on haematological parameters in healthy population," Medical Archives, vol. 71, no. 2, pp. 132-136, 2017.

[26] A. N. Burnett-Hartman, P. A. Newcomb, C. M. Hutter et al., "Variation in the association between colorectal cancer susceptibility loci and colorectal polyps by polyp type," American Journal of Epidemiology, vol. 180, no. 2, pp. 223-232, 2014. 


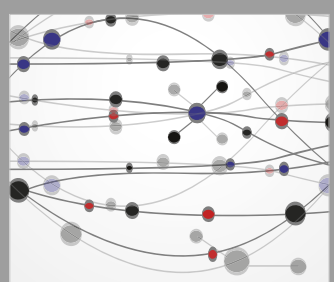

The Scientific World Journal
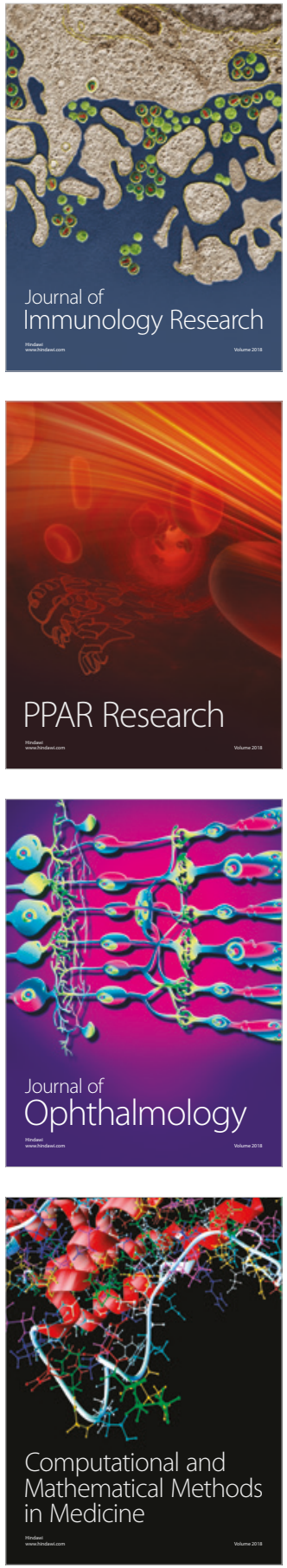

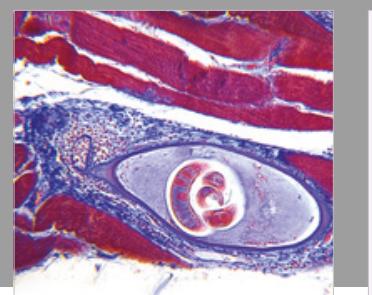

Gastroenterology Research and Practice

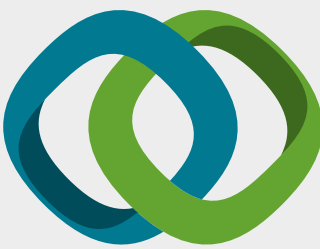

\section{Hindawi}

Submit your manuscripts at

www.hindawi.com
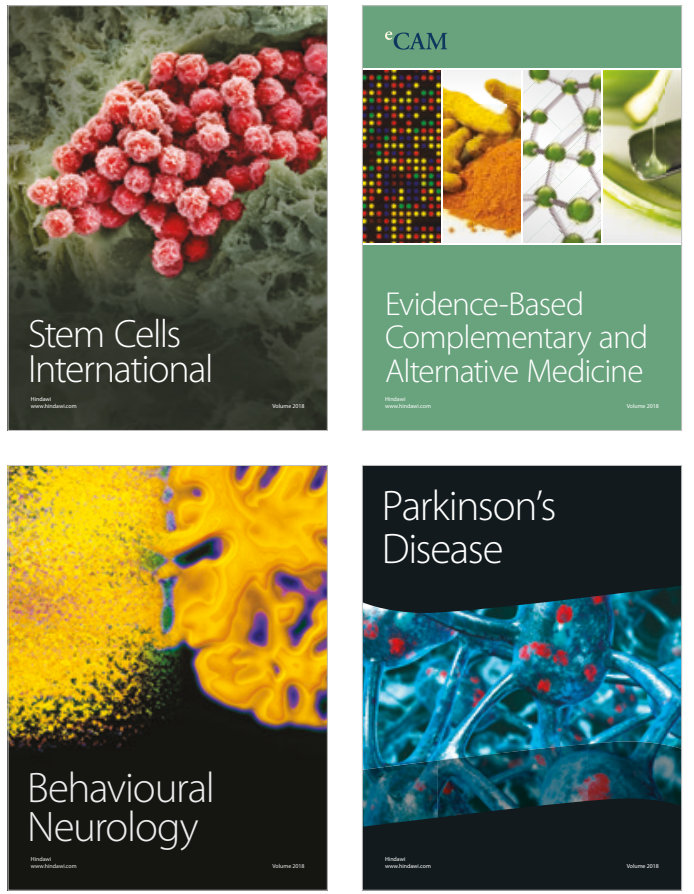

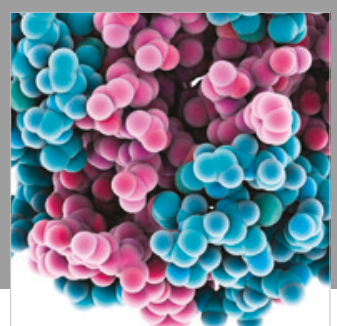

ournal of

Diabetes Research

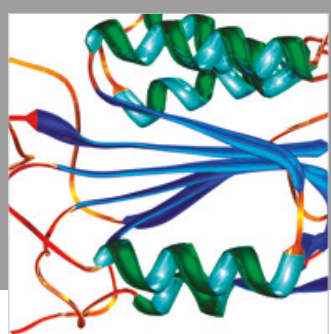

Disease Markers
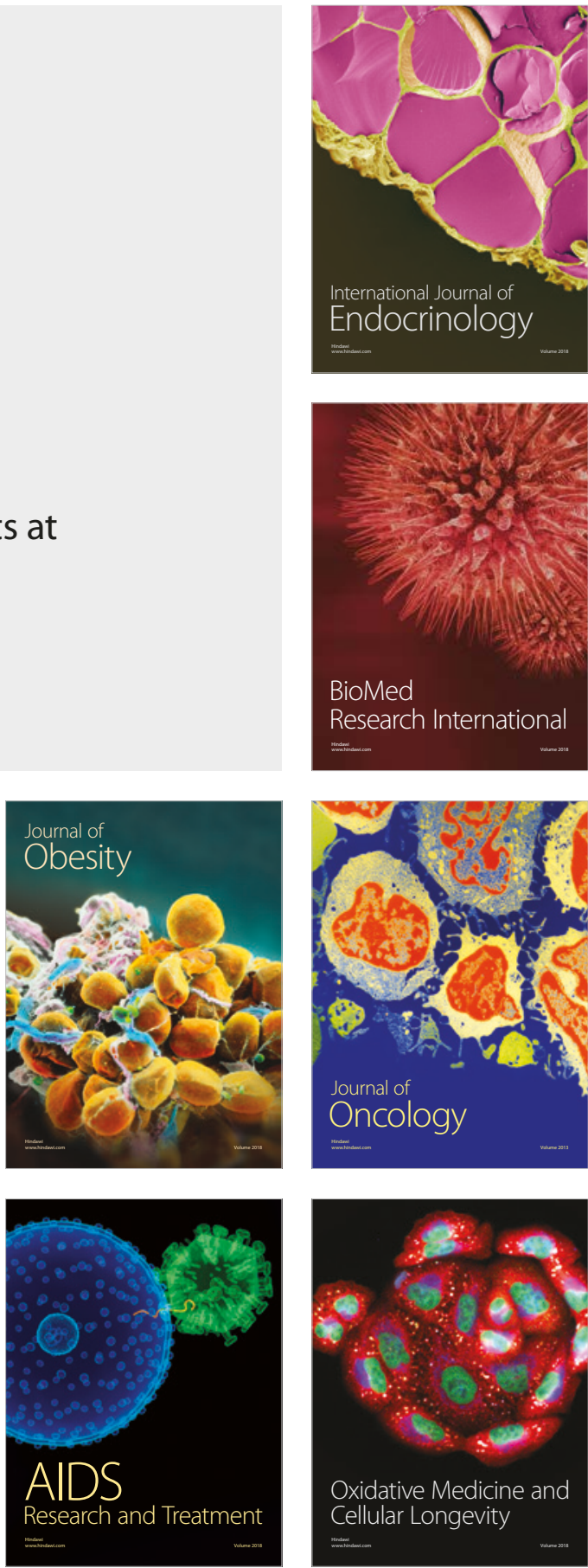